\title{
Smart Agriculture IOT with Cloud Computing, Fog Computing and Edge Computing
}

\author{
S. Nandhini, Shivcharan Bhrathi, D. Dheeraj Goud, K. Pranay Krishna
}

\begin{abstract}
Smart Farming could be a explained as a farming method which works on the thought process of a fashionable technology to increase the yield of the amount and quality of agricultural merchandise. IoT-based smart farming, a system solely made for the observation of crops in the field with the assistance of sensors and automating the irrigation system in accordance to our needs. Antique cloud-based system which uses mostly IoT models are inadequate to handle the traffic and the database of knowledge. So as to an extent it turns out to be lower latency, longer battery life for IoT devices, a lot of efficient money-based knowledge management, access to knowledge management and AI, ML IoT-EDGE based system is proposed or may be adopted. Edge for the IoT brings potential edges for several IoT deployments, as well as removal of interval in conjunction with geometric communications potency, compared to exploitation of the cloud to process and store knowledge. For example, several IoT processes will have a high level of automation at the sting leading to low latency for fast processing. The machine ifogsim is employed for modelling and simulating the sting based mostly on the IoT system which also includes the edge and the fog. The results of this method are to indicate that Edge computing based mostly IoT models are a lot of economical and extremely fast and may turn out and provide higher results when put next to different systems.
\end{abstract}

Keywords-IOT (Internet of Things) Cloud Computing Fog Computing Edge computing Smart Farming

\section{INTRODUCTION}

Agriculture, with its allied sectors is unarguably the most necessary support provider in this nation, Plenty of these practices cannot be done in the urban areas and hence are practiced inside the massive rural areas. Replacing human labor with machines may be a growing trend across multiple industries, and agriculture should not be any exception. Any other automations like the Driverless tractor such that wherever the tractor moves around the field on its own through an attached GPS system enabled within the tractor. Modern agriculture is an industry segment that has come not very far in terms of digitization and process automation.

Revised Manuscript Received on December 30, 2019.

* Correspondence Author

S. Nandhini, Assistant Professor, Srm Institute of Science and Technology, Chennai (Tamil Nadu) India.

E-mail: Nandhins1@Srmist.Edu.In

Shivcharan Bhrathi, Srm Institute of Science and Technology, Chennai (Tamil Nadu) India.

E-mail: shivcharan077@gmail.com

D. Dheeraj Goud, Srm Institute of Science and Technology, Chennai (Tamil Nadu) India.

E-mail: dulamdheeraj12@gmail.com

K. Pranay Krishna, Srm Institute of Science and Technology, Chennai (Tamil Nadu) India. E-mail: pranaykrishna71@gmail.com

(c) The Authors. Published by Blue Eyes Intelligence Engineering and Sciences Publication (BEIESP). This is an open access article under the CC BY-NC-ND license (http://creativecommons.org/licenses/by-nc-nd/4.0/)
A wide range of new technologies for crop and livestock management related to sensors, GPS attached to tractors, image processing and machine learning are now available for use in daily operations in our very own palms. Edge computing not only allows incoming data to be analyzed close to the source with a less noise (Which is to be cleaned with data mining techniques),it makes it easier for refined data - or the results - to be sent over narrow-band networks and also makes it easier to follow necessary protocols. This opens up a array of new business opportunities. When the sensors are introduced the data is all collectively recorded automatically without any human interference. These sensors are only used in the most obvious fields and are not used much in agriculture. For example, we're able to detect which animals or birds are active or dormant, ill or is it hale and healthy, or submissive or dominant in order to adapt elements of their care, including special treatments, living conditions, medication and nourishment. Therefore, when the treatment can be done for the animals and living fauna then why not can it be used in the case of flora. There is an implementation or an idea which is put forward already in countries like Sweden and Finland by a group of people called the Imagimob. This is but not sadly implemented in the agriculture capital of the world India.

\section{CLOUD COMPUTING}

In the easiest terms, cloud computing means accessing and querying data and functions whenever necessary and providing with low redundancy and this all happens over the web instead of your computer's memory. The cloud is solely a pathway from the online web to the people who use them. Cloud computing could be defined as a mix of technologies that structure a network for the giving of computing services. It desires hardware for infrastructural programs and software system packages to deliver the on-demand services over the web. There square measure differing kinds of cloud computing services on the market to suit fully totally different needs. whereas some cater to individual users UN agency would like to store photos, documents, and videos, others square measure destined for firms that need intensive platforms to develop IT applications

Cloud computing involves in operation pc functions and capabilities in an exceedingly service access via an Internetbased platform. It's unremarkably used for information computing, storage, and management that takes place off an enterprise's native machines. firms all round the world deem the cloud to manage their information aboard making and corporal punishment workflows.

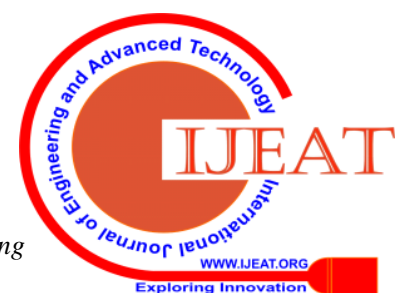


However, the idea of cloud computing has solely been around for simply over a decade, and plenty of enterprise leaders don't quite perceive what the cloud truly is.

Crop-related info-It can capture data related to all crops mature at intervals the recent past, and so can facilitate farmers produce alternatives on what to grow next. Weather info-The cloud can store region-specific weather data and any as a result of the forecast for specific durations. Soil Information- Crop-related decision-making depends totally on soil data too. excluding the profile, it may give a trend of soil within the past, which can facilitate in predicting the trend within the future. observation Growth: the growth of assorted crops is also monitored in many regions; and at regular intervals. this permits growth patterns to be compared with past growth patterns. Farmers' DataRegion-wise farmer information may be captured, monitor and study the involvement of native farmers.

This can facilitate at intervals the identification of core agricultural areas, that unit helpful for policymakers whereas framing their strategies.

Cons

Security and privacy- Any discussion involving info ought to address security and privacy, significantly once it involves managing sensitive info.

Vulnerability to attacks -In cloud computing, every half is likely accessible, however since cloud computing is formed as a public service and it's simple to run before you learn to run.

Restricted management and suppleness to variable degrees cloud users have restricted management over the perform and execution of their hosting infrastructure.

\section{Fog Computing}

Cloud computing is a good technique which can be used along with IOT devices and there are various applications but they cannot be used with various IOT devices dues to which fog computing is used. Fog has a wide range of uses with IOT devices. They can be used for industrial purposes and also they are various applications with sensors where the data is collected and communicated faster and securely. The data that is collected analytics is done on them so that farmers can take better decisions. Fog computing helps in reducing the information measure required and in turn reduces the communication between the sensors and the cloud due to which fog is very good in data communication when compared to the cloud-based platform. Fog computing has various frameworks within them due to which there are various ways in which data can be collected and processed, the user or the organization can use the appropriate framework required for their purpose and get the desired. For many fog applications, knowledge Is collected and processed quickly. Fog computing produces low latency and better network and data connections between the devices produce the best required output after the data is collected and processed. The fog design successively helps in reducing the quality and the quantity of the knowledge gained. Fog computing may also be utilized in situations wherever there's no information measure affiliation to acquire knowledge, so the process of the data collection and data processing must occur close to the IOT device which does the process and gives efficient results to farmers.
Whenever we need low latency and low cost, fog computing is used. It can act in real-time on the incoming data and works within the limit of available bandwidth. Fog computing provides a better quality of performance and QoS service and also in terms of latency, power consumption and data traffic over the internet and iot device when compared to the cloud platform. Moreover, with the assistance of the analysis generated through the FOG computing suggestions will be made for fertilizers \& pesticides \& which crops to be grown in the farm. Drawbacks or disadvantages of Fog Computing are that mistakes in the algorithm can result in the data being hacked. So the security of the data is an issue. Other security issues are IP address is spoofed and middle attacks are done and the data can be stolen before it is stored in the cloud. To achieve high knowledge consistency within the fog computing is difficult and needs a lot of efforts. Fog computing can understand international storage thought with infinite size and speed of native storage however knowledge management could be a challenge. Trust and authentication are major concerns.

\section{Edge Computing}

Edge computing is similar to that of fog computing the only difference is that in edge computing the process occurs on the edge of the IOT device and the results are produces immediately without the need to go to the cloud and return back with result. Similar to the fog computing the edge computing also have various frameworks from which the user or the organization can choose from. Generally, in the cloud or the fog-based platform the devices would collect the data from real time and send it to the cloud to process them and get the desired result but in the case of edge computing the data is collected and processed then and there. Due to which there is no problem for the data security as the data is not communicated to the cloud due to which the data cannot be hacked as in Fog computing and also there is no problem of network traffic as the result is produced there itself that is even if the network traffic it does not affect the edge computing. Edge computing allows smart devices to respond in real time data instantly because the data is collected and processed the the edge of the device only due to which faster results are produced without any lag. Edge computing has more advantages when compared to the other platforms like cloud and fog computing as the response time is low, low latency and no problem of network traffic and they have better communication between the device and the cloud and it occurs in a faster rate. The main aspects on why Edge is better for the IOT are-Low Latency, Longer battery life for IOT Devices, More efficient Data management. When the data is collected in the web with the help of sensors the data would have to be pre-processed and also cleaned of any illogical substances present in it. This is where edge computing and Edge AI solution comes in. Edge computing not only allows incoming data to be analysed close to the source with a minimized complexity, it makes it easier for refined data - or the results - to be sent over narrow-band networks. 


\section{LITERATURE SURVEY}

Sucharitha. V, Prakash. P, Ganesh Neelakanta Iyer developed a IoT device with fog computing for smart agriculture called AgriFog- A Fog Computing based IoT for Smart Agriculture the techniques used were Fog computing with IoT devices and the simulator was ifogsim. The pros of this review was The outcomes of the simulations are assimilated to discern the performance highlights of the fog based deployment over the cloudbased deployment. And the cons were Highly latent in nature and less efficient.

Tomo Popovic, Nedeljko Latinovic, Ana Pešic, Žarko Zečevic , Božo Krstajic , Slobodan Djukanovic developed a report for the system architecture of the IoT device based platform. The techniques used were IoT devices on Arduino along with the Raspberry Pi connected to the PC. IoT protocols, CoAP Languages R, python. The pros were that the solution obtained will be used for the purpose of data collection with redundancy which is then used for the prototyping of the analysis functions. The cons were Extremely Insecure and high risk of data stealing.

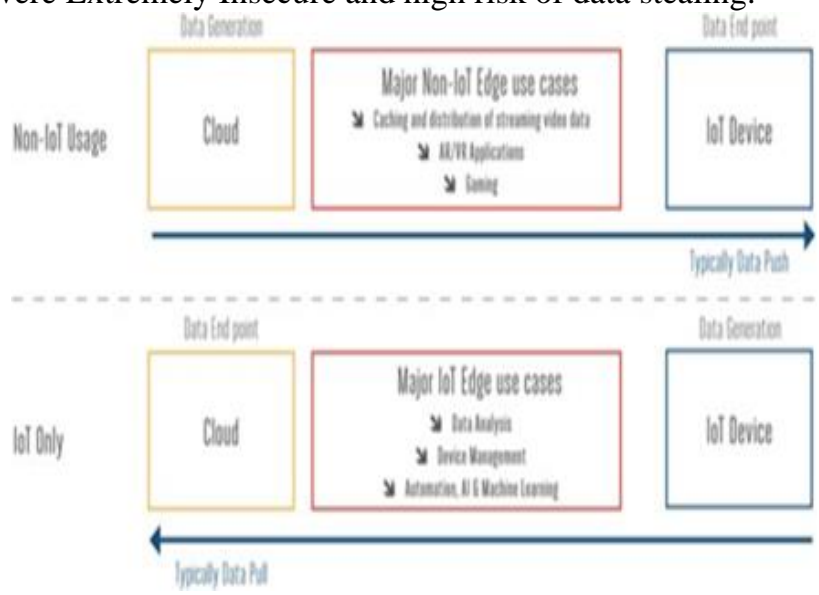

Figure (1): Data Flow in Cloud IOT

Tamoghna Ojha , Sudip Misra, Narendra Singh Raghuwanshi developed a sensor cloud for agriculture. The techniques used were sensor-cloud framework by considering specific challenges. The pros were that the specific challenges that are faced are solved by mathematically devised virtual technique which underlies the sensor framework and give desired solutions.. The cons were A highly complex system which requires complex calculations.

Mahammad Shareef Mekala, Dr P. Viswanathan developed a journal for IoT with cloud which shows how the cloud computing is used for the purpose of smart agriculture. The pros were that the devices cost less and the power consumption by the device is also less when compared to the platforms. It also provides QoS platform which makes the device give an efficient performance and they can be easily understood by the farmers so it is easy to use. The cons were High space complexity.

Shanhe Yi, Cheng Li, Qun Li developed a report for IoT with fog based computing called A Survey of Fog Computing: Concepts, Applications and Issues. The techniques used were Fog based computing which results in faster data communication and better security and better latency when compared to the cloud computing which is implemented on IOT and edge devices. The pros were that the techniques used has better communication of the data that is being collected, they have a lower latency when compared to the cloud-based platforms. The cons were High latency and decreased efficiency.

Prem Prakash Jayaraman, Ali Yavari, Dimitrios Georgakopoulos, Ahsan Morshed and Arkady Zaslavsky developed a IoT device which is used in smart agriculture where the IOT device learns for the experiences and the lessons learnt and from the all these experiences and lessons, proper decisions are taken by the IOT device for smart agriculture. The techniques used are that they built an device which was issued for the purpose of data collection, analytics and the data visualization to take better decisions . The pros are that they developed a unique approach which gave efficient results at the real time. According to the user commands a real time response is received which is used to take better decisions for smart agriculture. The real farming data was used for this purpose and the elasticity and the scalability of the approach was justified

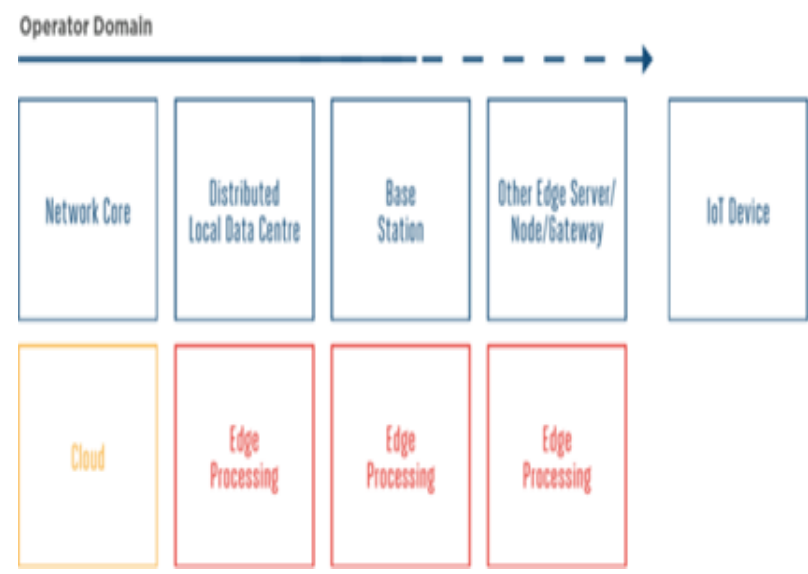

Figure (2): Data Flow in Edge IOT

Bonomi, Rodolfo Milito, Jiang Zhu, Sateesh Addepalli developed the role of fog computing in IoT devices and their advantages. The techniques used were the connection between the iot device and fog Computing suing necessary sensors. The pros are that they developed a brief journal which was used to deliver responses at the edge of the network. The sensors such as the mqtt protocols and various other sensors were used for the purpose of the data collection and secure the data in the cloud.

Jnaneshwar Das Bonomi, Rodolfo Milito described about the devices which was developed for the purpose of monitoring and data collection. The techniques used are methods to improve the smart agriculture by introducing light weight a methodology for precision agriculture consisting 3-D imaging system which is capable to be used on flying machines. The pros are that the journal gives an efficient system which produces efficient results in automated monitoring device which is used to take better decisions for precision agriculture and providing sustainable production. The design is very simple and compact due to which is easier to understand and the farmers can understand easily and use this approach.

Mohan raj, Tamoghna Ojha developed an automated device which is used for field monitoring they tell us how these devices and automations are used in agriculture.

Published By:

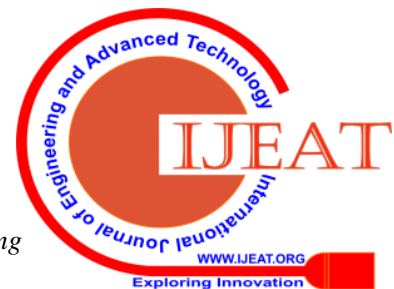


The techniques used are that there are various steps of monitoring like remainder of the plant, plant growth is monitored, the irrigation is planned accordingly, and the climate is checked at various intervals to check for the occurrence of disasters, which does not result in the crop loss. The pros are that due to the study we understood the availability in the current system and that they can be easily understood and the process is very simple and the system architecture is simple and compact.

K. A. Patil, N. R. Kale developed for smart agriculture called A Model for Smart Agriculture Using IoT. The techniques used that they developed a efficient smart agriculture model which was used along with the ICT. The pros are that they give a brief knowledge of the local agriculture information at various conditions. From this approach we can develop various models for the smart agriculture with iot device which can help the farmers take a better decisions and get better crop profit.

\section{ANALAYSIS}

Agriculture is one of the most important fields in any part of everyone's society. The concept of farming although has not been modernized in a vast manner in terms of deciding what crops to yield at what time. The method which is scarcely used now is Cloud computing and is used scarcely because of the cons it has. To overcome this and provide a better way of implementing IOT (Internet Of Things) with improved complexity ratios and secure manner we introduce a method called as edge computing.Smart agriculture, also known as precision farming, helps farmers to increase yields by using minimal resources such as water, fertilizer and seeds. Farmers may begin to understand their crops on a micro scale by installing sensors and mapping fields, conserve resources, and increasing environmental impacts. Thus, we can determine the minimum resources required for a given land or area at least as an approximate value by determining all the necessary data i.e. $\mathrm{pH}$ value, soil water content and capacity. Once farmers were able to map their crop fields accurately, they were only able to monitor and apply fertilizer and weed treatments to areas that needed it.

\section{ARCHITECTURE DIAGRAM}

The given architecture diagram can be divided into several different modules. The diagram shows how the process of edge computing works and also how it is different from the process of Cloud or Fog computing. There are basically a number of modules in this given architecture diagram. The first module is the connection between the Farmer and the Sensors. There are different types of sensors and these sensors and these identify and collect the data according to the predefined and set values given to it while setting it up. These sensors collect live data for a given amount of time and then send it to the next stage.The next module or next stage is when the data goes from the sensors to the system server and then to the router simultaneously. The system server is like an admin system which keeps all the data stored as a copy for the future references and other details. The system server sends the data to the router now for all the transfer of data to the network cloud. This router has to follow several protocols for the sending of data because it is

an extremely complex process and predominantly CLNP (Connection- less n/w protocol) and State Routing Protocol along with (SRP) Media Transfer Protocol (MTP) is used. There is also a protocol called as the gossip protocols which is used for data security up to a certain extent. The routing of these data to multiple protocols cause the data to become extremely insecure. This therefore provides a major drawback to cloud and fog computing.

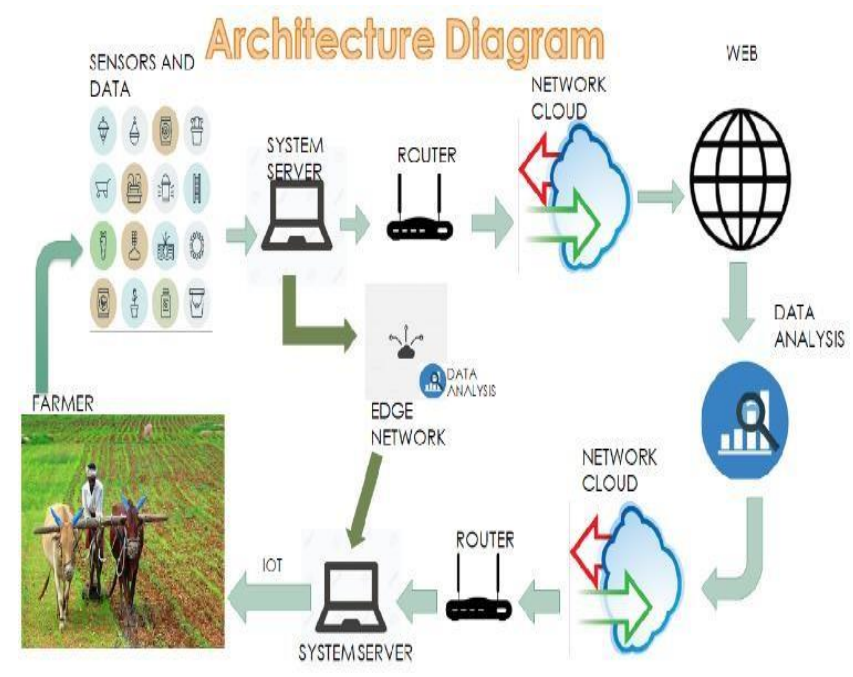

Figure (3): Architecture Diagram

The third phase would be the pre final phase which is extremely important and is called as data pre- processing. In this process the data is cleansed and removed of any possible noisy data. The noisy data is cleansed by methods like removing the tuples and putting the mean value method. This involves the use of Data mining, after the data is preprocessed (i.e.) cleaned the data is sent for data analysis. In this step the data gets pasted in a web kind of environment and this is now mined for Interesting patterns based on prefixed values. The data is then further calculated i.e. for example if there is soil data collected then then predefined values of soil would be checked and the best case for which crop could be calculated is predicted and then sent back the same way it came which is through the router and then through the data cloud and then finally the farmer's application. This is how the cloud computing works. The method that is being proposed in this paper is Edge computing in the place of cloud computing- In the case of edge computing which is also given in the same diagram on how it is better than the Cloud computing process. Edge computing requires only 2 steps and that will be both to and from the user.The data first gets sent by the sensor to the System server and then from there in need not get transferred to the system cloud and so on. When the data is received by the router then immediately the process of data analysis starts happening. The Data pre-processing happens during the transfer of the process and it happens in the network cable. Once it reaches a particular point and the data is seemed to be ready then the data will be automatically sent back and the source is not very far because all these has happened in the edge node which is close to the source. 
The data is now sent back with extremely low latency and also in a very secure manner. The data when getting transferred in the cloud computing process can be stolen or distorted due to the complex protocols it follows.

\section{Agricultural Sensors}

Location Sensors use GPS satellite signals to determine latitude, longitude and altitude within feet. To locate a position, a minimum of three satellites is require. Optical Sensors light is used by optical sensors to measure soil properties. Across near-infrared, midinfrared, and filtered light spectrums, the detectors measure different light reflectance frequencies. It is possible to place sensors on vehicles or aerial platforms such as drones or even satellites. Electrochemical sensors provide key farming information: $\mathrm{pH}$ and soil nutrient levels. The electrodes of the detector operate by detecting different soil ions. Mechanical sensors measure the compaction of the soil or "mechanical resistance," using a device that penetrates the soil and measures resistive forces using load cells or pressure gages. The permeability of soil air is measured by airflow sensors. In movement, calculations can be done in single or liquid locations. All of the above information is collected together and then automatically processed at the source as the data is not expected to be sent to the server, which reduces reliability and is also extremely timeefficient.

In addition to smart sensors for data collection and connected devices, edge analytics includes data storage hardware and software systems, data preparation, learning algorithms and processing algorithms. In each of these areas, decisions need to be made with regard to data collection, data preparation, selection of algorithms, continuous training of algorithms, deployment / redeployment of models, etc. Store energy management at the edge also plays a key role. Edge computing provides services for low latency, efficient bandwidth and resilient end users. Using this service, users get a latency benefit for those who are away from the data centers. Edge computing provides extended capabilities to deploy application in the edge networks of traditional data centers. Solving problems for large and small farms and helping farmers fulfil everincreasing food demands are not the only options that can be offered by intelligent, precision agriculture. Smart farming offers a number of other benefits, such as:

Reducing fuel consumption and reducing emissions of carbon dioxide. Through maximizing the use of nitrogen fertilizer, increasing nitrous oxide emitted from soil. Reducing pesticide use by defining criteria for fertilizer and pest control. Eliminating loss of nutrients by control and soil health management. Operation of soil compaction by reducing the traffic of machinery Maximizing the performance of water use.

\section{CONCLUSION}

The IOT is revolutionizing the world by providing multiple solutions and also giving many different methods such as the Cloud, Fog and Edge computing. The cloud has been there in this world for a pretty long time and the new modernizations are the fog and the edge. Both of them are similar but the only difference between both is that the edge is faster and higher latent than the fog and cloud. Edge computing is already being used in daily livestock and in other places. Agriculture although has not been modernized and it still follows antique methods. Edge computing if implemented could revolutionize everything and provide a great boost in the agricultural sector. Processing the data at its source is called as edge computing and this Is extremely useful in remote areas and therefore is perfect in the case of agriculture.

\section{REFERENCES}

1. Prof. K. A. Patil, Prof. N. R. Kale - A Model for Smart Agriculture Using IoT

2. Tamoghna Ojha a,b,爪, Sudip Misra a, Narendra Singh Raghuwanshi b - Sensing-cloud: Leveraging the benefits for agricultural applications

3. Alexandros Kaloxylos a,b, $\Uparrow$, Robert Eigenmann c, Frederick Teye d, Zoi Politopoulou e, Sjaak WolfertfClaudia Shrank g Markus Dillinger c, Ioanna Lampropoulou a, Eleni Antoniou e, Liisa Pesonen d,Huether Nicole g, Floerchinger Thomas g, Nancy Alonistioti a, George Kormentzas e - Farm management systems and the Future Internet era

4. Alexandros Kaloxylos a, $\Uparrow$, Aggelos Groumas b, Vassilis Sarris b, Lampros Katsikas b, Panagis Magdalinos bEleni Antoniou c, Zoi Politopoulou c, Sjaak Wolfert d, Christopher Brewster e, Robert Eigenmann f,Carlos Maestre Terol - A cloud-based Farm Management System: Architecture and implementation

5. Mohanraj I*a, Kirthika Ashokumarb, Naren Jc - Field Monitoring and Automation using IOT in Agriculture Domain

6. Mahammad Shareef Mekala, Dr P. Viswanathan - A Survey : Smart Agriculture IoT with Cloud Computing

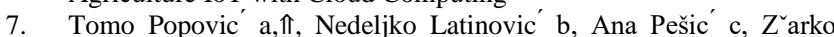
Zec `evic' d, Božo Krstajic d, Slobodan Djukanovic' - Architecting an IoT-enabled platform for precision agriculture and ecological monitoring: A case study

8. Shanhe Yi, Cheng Li, Qun Li - A Survey of Fog Computing: Concepts, Applications and Issues

9. Lihua Zheng a, Minzan Li a,*, Caicong Wub, Haijian Ye a, Ronghua Ji a, Xiaolei Deng a,Yanshuang Che a, Cheng Fub, Wei Guoa Development of a smart mobile farming service system

10. Flavio Bonomi, Rodolfo Milito, Jiang Zhu, Sateesh Addepalli - Fog Computing and Its Role in the Internet of Things

\section{AUTHORS PROFILE}

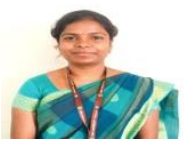

MS. S. Nandhini, Assistant Professor at SRM Institute of science and technology.

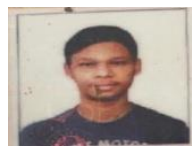

Dheeraj Goud D., $3^{\text {rd }}$ year cse undergraduate pursuing B.tech SRM Institute of science and technology.

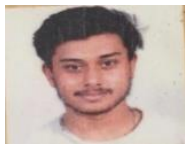

K. Pranay Krishna, $3^{\text {rd }}$ year cse undergraduate pursuing B.tech SRM Institute of science and technology.

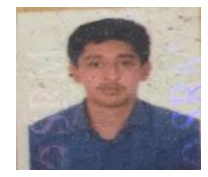

Shivcharan Bharathi, $3^{\text {rd }}$ year cse undergraduate pursuing B.tech SRM Institute of science and technology. 